\title{
Discussion on the Symphonic Question of Twelve Muqam Uygur Art from Xinjiang, China*
}

\author{
Nuermaimaiti Yimamu \\ Institute of Uighur Language and Culture \\ Northwest Minzu University \\ Lanzhou, China 730001 \\ Kashgar Art School \\ Kashgar, China 844001
}

\author{
Adila Aimaiti \\ Institute of Uighur Language and Culture \\ Northwest Minzu University \\ Lanzhou, China 730001 \\ Kashgar Art School \\ Kashgar, China 844001
}

\begin{abstract}
The Muqam Uygur Art from Xinjiang China which carries ancient national culture, is the crystallization of people's wisdom, a precious heritage of national culture, as well as the real reflection of Uygur people from hundreds of thousands years. Its unique art value and cultural connotation is considered as World's intangible cultural heritage, which not only belongs to a nation, but the whole world. In order to get more widely attention and follow up from the society, we need to carry on this exquisite national culture and protect its variety. This article aims to discover the symphonic problem existing in the Twelve Muqam Art, with firstly illustrating of its uniqueness on the historical cultural connotation and characteristics, also its dancing cultural features and music form, together with the characteristics of its historic significances, varieties and improvisations, etc. mainly to elaborate if the Twelve Muqam Uygur Art should be symphonic by comparing the differences and commonness between the European Symphony and the Chinese Twelve Muqam, in order to give a final corresponding thinking with the best mentality and great hope, we wish a better development and inheritance of Muqam Uygur from Xinjiang, China.
\end{abstract}

Keywords—symphonic; Uygur; historic significance; Muqam

\section{INTRODUCTION}

The Muqam Uygur Art from Xinjiang China as an important national intangible cultural heritage created by the Uygur and other minorities from the Chinese western area. The Twelve Muqam Uygur Art from Xinjiang China consists the art of Naghma, Dastan, Mashrap, with strict artistic structure, such as cultural sites, utensiles, musical instruments, folkloric activities, traditional knowledgerelated are also part of the its important component. Various forms of performance and deep cultural connotation empower extremely high academic and artistic value to it,

*Funded project: year 2017 national social science fund of art department youth project — stage research outcome of "Intangible cultural heritage of Twelve Muqam academy development" (approval no. 17CH219) ; year 2015 national social science western project — stage research outcome of "A special study of Uygur folk oral Poetics" (approval no. 15BZW196 ) ; year 2018 Northwest National University Central College essential science research fund for post-graduate project - stage research outcome of "Music Research of Uygur folk love narrative poem Gherip-Senem" (approval no. Yxm2018003). and it covers the production lifestyle of oasis nation. In 2005 , the Muqam Uygur Art from Xinjiang China was selected to the "List of representative works of intangible human cultural heritage" with honor, which brought wide attention worldwide. However, facing the concept of symphonizing the Muqam together with orchestral ensemble, whether to bring this intangible cultural heritage to the road of orchestra is a big question.

\section{COMPARISON ON THE ELEMENTS BETWEEN} EUROPEAN ORCHESTRA AND TWELVE MUQAM UYGUR XINJIANG, CHINA

\section{A. Differences}

Performance form: singer or performer in Mu Card Mar of Twelve Muqam can sing or perform simultaneously, after $\mathrm{Mu}$ Card Mar, singer can directly enter phase Naghma or Dastan or Mashrap, and therefore it has very high level of improvisation. When performing an orchestra emsemble, it lacks of improvisational possibilities because of orchestra's strict structure and creativities of composers. In many situations, orchestra requires to perform the whole chapter. Besides this, conductors are mandatory and hold important position, while performing Muqam, there is no conductor.

Tonality: three-quarter temperament is very common in Twelve Muqam, tones can move up and down together with fixed tone class which has great impacts on the music style. For example, special symbols can be seen and used to highlight the characteristics of each tone. But in orchestra, each music class is fixed and divided evenly into Ikkis temperaments. Tones cannot be moved up and down and no live notes available unlike the tonality of Muqam.

Arrangement of orchestra: in Muqam this folk music, Kashgar's Twelve Muqam is very classic, thus this is used by its national musical instrument along with cello. In the 17 th century, orchestra is rising with the development of opera. Therefore, symphony performed by the orchestra use mainly the musical instrument from Europe. Dap, Nagra, Tashi, Shapayee, Nai, Sunai, Dutar, Tanbul, Jewafu, Qiang, Karen, Aiczech Republic, Shatar, Husitar are the main musical instruments used by Twelve Muqam ; And Clarinet, Oboe, Trombone, Violin, Viola, Cello, Timpani, Harp, Flute, 
Piccolo, Trumpet, Bass are mostly used by orchestra. From different musical instruments, we can see a big difference between Muqam and Orchestra in sound effect and the manner of performance. But in structure Violin and Aiczech Republic have certain similarities; however they are still 2 different instruments in shapes. In addition, Trombone, Trumpet, Flute, Piccolo from orchestra are quite distinct from Sunai and Nai such wind instruments.

Rhythm and beat feature level: Twelve Muqam has great contrast with symphony through the varieties of its applicable beats and unique form of rhythm. Twelve Muqam is rich and colorful in the form of beats, such as the regular $4 / 2,4 / 3,4 / 4$ beat, as well as the complexed $8 / 3,8 / 5,8 / 6,8 / 7$, $8 / 9,4 / 5,4 / 6$. While symphony is mostly regular in beats but its rhythmic transformation is also very characteristic. In the form of rhythm, the Mu Card Mar's part of "Naghma" is loose, afterwards reach the parts of Tai Zhi, nun ha' $s$ rhythm. The part of Dastan's form is middle rhythm, the form of "Naghma" is mid or slow rhythm, and Mashrap's form is happy and fast rhythm. Obviously, the whole rhythm of Muqam is strong and happy, from slow to fast pace, to push the mood all way to the climax then to the end. While symphony has 4 chapters in rhythm, they are fast, slow, small, and fast pace as the final chapter, overall speaking, the rhythm of symphony is quite fast-paced.

Headline: each component has its headline and name in Twelve Muqam. For example, during the part of "Naghma", it includes Mu Card Mar, Tai Zhi, Nun Ha etc 10 to 15 tunes, "Dastan" has 3 to 10 tunes and interludes, and "Mashrap" has 1 to 3 tunes. Afterward, the whole Muqam ends in slow, irregular and free beats. Generally speaking symphony doesn't have headlines, but can be created based on the content and your own willingness. Like "My Fatherland" by Bedrich Smetana, created during the period of nationalism music, consists of six independent works, of which "Vltava" is the headline of the 2nd work. Additionally, Hector Louis Berlioz, the "Father of Composer in Symphony", his representative works "Fantacy Symphony", "Harold in Italy" are also like this. In most cases symphony doesn't have headlines that reflect the detailed content of musical works.

History: during Mannheim period, symphony starts to rise in Europe. Such a music genre is created based on orchestra ensemble, with the leading of Franz Joseph Haydn, "Father of Symphony", it has a very strong professionalism and it is a result of many composers' efforts. In comparison, Muqam is formed during 16th century, Ming Dynasty, such music genre is created based on collecting from folk long poems, Mashrap music and traditional music, being changed and transformed in many ways, today Muqam slowly becomes in good shape during the historical process.

Adjustment: Music system from Persian-Arabia, Europe and China is a standard normally used adjust music. After many times' arrangement, evolution and collection, Muqam absorbs other nations' art characteristics and musical outcomes, and has become very unique in geography and era. In other words, Muqam is a combination of music system from Persian-Arabia, Han majority of China mainland, local traditional Uygur and Europe. While symphony use 12 average rhythm-based 7-tone musical adjustment system. During the creation of symphony, composers adjust frequently melodies; but the major changing manner in Muqam is the change of rhythm and beats.

Content in performance: sometimes symphony is accompanied by lyrics, that's because it's an instrumental music. Twelve Muqam Uygur has comprehensive characteristics, including lyrics, music and dance, three in one. Besides, Muqam is a type of music very expressive and narrative, easy to express emotions. The public can directly feel the emotions brought by Muqam when appreciating parts of Muqam music, which proves Muqam has direct and concrete features. When listening to symphonies, it's a bit difficult to understand without singing or dancing. If people want to feel what it means, they have to refer to the style of music, which makes symphonies more indirect and abstractive.

\section{B. Similarities}

From the aspect of whole structure regulation: they both have significant scale and represent big-scaled music works. Most of them have formed the Twelve Muqam Uygur music. Its melody, speed, and rhythm are very changeable, from slow to fast. Performing each part can bring people rich and happy feelings with its scientific and flexible technics. Twelve Muqam can add Dastan, Mashrap and Naghma after $\mathrm{Mu}$ Card Mar, alternatively can sing and perform independently with the same sequence to perform the whole entity. This characteristic can last long time depend on the detailed situation, place, condition and environment. Therefore, it's obviously to see Muqam can perform certain parts individually and flexible enough to master. However symphony normally must perform the whole chapter, and sometimes can perform certain parts depending on its concrete condition and situation. Not only that, short, free, happy and strong characteristics exist in the part of Mashrap of Muqam with bright and lively rhythm, and the last chapter of symphony also has vibrant and inspiring features, which shows the similarities in both music.

Development path: both of them are developed simultaneously together with the history of human being and have long historical background, which are considered as two very important music systems with great impacts on the world-class music art, although they were created from different geographies and eras. It doesn't matter where the artists are coming from, European composers or Uygur folk artists, they can combine perfectly the music with literature and other art elements from different nations and bring their talents to those 2 genres of music works to better show the development path of national music culture profoundly, meanwhile make them become classic works of national music culture with deep accumulation of national culture. Over a long period of time, European symphony artists serve the royal families exclusively, which are followed and welcomed widely by the upper class. Similarly, the Twelve Muqam also experienced the same development process, from royal palace to folks. 
Performance of band: from the composing way of speaking, both of them are composed by blowing, hitting, pulling strings, and plucking string instruments. For example, Ghijak from Twelve Muqam consists of structures of bass, tenor and treble. The viola class from Symphony consists of 4 structures, small, medium large and low bass. In theory of making, some musical instruments also have certain similarities, which are both used by their national musical instruments, one from Europe, and the other is from Uygur.

\section{Reflections}

In Uygur, Twelve Muqam is the classic music divertimento, and Symphony as an elegant music, is highly praised by the kings and nobles. They were created in different historical backgrounds, thus have different ethnic style and epoch characteristics. Both have certain way of music creation and charms by considering their different form of historical backgrounds. Their creation and development have influential impact on the development of future world's music art. Currently the European symphony is well-improved in terms of band's structure and music composition, and many excellent composers stand out along with its development process, who are devoted to blend in big amount of national elements in order to demonstrate the national artistic characteristics by western musical instruments. Through the base of creation principles, make constantly self-breakthrough of music, and by changing the content and structure of bands, adapt the needs of the appreciators' taste.

In music art of Uygur, Kashgar's Twelve Muqam is the most representative classic music. Due to the impact of modernization and lack of successors, Twelve Muqam as the important cultural carrier of Uygur nation is in danger. Hence, the focus of researcher currently is how to protect and inherit, as well as a good reference for the future of Twelve Muqam Uygur and prosperity historical process of symphony.

\section{ANALYSIS ON THE SYMPHONIC QUESTION OF TWELVE MUQAM UYGUR ART FROM XINJIANG, CHINA}

"Live Tone" — 3/4 beat exists in Twelve Muqam's melody, with its original expressive form of vocal, instrument and dancing, three-in-one, together with clusters and beats of music, which allow the singers and performers to bring the unique art value of Twelve Muqam. With recognition of national culture and religion and its covering on the dancing, rap, theatre, literature and music so on, it gets the attention and recognition of the world and has "Live Fossil" such significant meanings. But facing our country's politic, economic and cultural progress and the development of information and scientific technology, the way and habit of people's living as well as their aesthetic consciousness are changing, which put Muqam in danger. The younger generation is indifferent to traditional festivals, neither like nor recognize its cultural value, which make Muqam communities slowly collapsing. All these factors are not favorable to protect and carry on Muqam art. Therefore, facing this endangered art nowadays, our priority is to "protect" and "inherit" instead of innovating or developing.
China points out: "Through social and school education such approaches, this intangible cultural heritage can be inherited and developed as a living cultural tradition in the relevant communities, especially among young people". But in current realistic situation, the younger generation lacks knowledge on the self-protection awareness, understanding, recognition and comprehension over the level of our local intangible cultural heritage. Even the reorganization of certain experts varies in the aspect of social culture and historic value of the intangible heritage.

Obviously, the key of western music is the composer, while in our case is the performer and singer, which is the biggest difference compared to the western music system. The current artistic achievements condensed in our country is based on the unique humanities and scenes of each period, as well as the development of each artist's independent personality and character passed by words of mouth. In Twelve Muqam Uygur music, you can get to know the local people's life and social style meanwhile enjoy the music and the dancing. Improvisation is the crystallization folk artists' wisdom during the whole music art performance, expressive in emotions, a place where the charms and essences of traditional folk art can be found. Improvisation, creation, and self-entertainment are filled in Muqam music, plus the tone, temperament, performance manner and structure of each instrument are extremely individualistic.

Muqam art as an intangible cultural heritage, disregarding whether the concept of symphonic development of national music is right or wrong, it is a way to discard the advantages and remove the uniqueness of national music, which is not an appropriate way to go. Bringing the impact on the audition and visibility to the crowds is the key point of symphony, which requires the vibration of instrumental structures, the intermingled integration of voice parts, and the unity of tone and temperament, and all these are just not the advantages of national folk music. Imagine one day, "Fujian Nanyin" has developed into a form of national orchestral ensemble, simply to cater to the trend and expand the structure; Likewise, it's going to be very hard to imagine the chor and long tune from Mongolia have been innovated and composed by professional composers to a 100-people chorus.

Since the reform and opening-up, China and regional leaders carried out rescue work in time, they have recorded, written and sorted precious materials left by musicologist such as Tongshu Wan who saved the Twelve Muqam in the only existing place, Xinjiang region. Relevant department have stepped up efforts to protect the Muqam after the successful application of heritage, making great efforts and contributions to the aspects of major, education, text, ecology and media inheritance. For example, opening Muqam major in Xinjiang Art School, supporting the successors, holding seminars and building inheritance center, etc. Xinjiang Muqam art group was founded in 1980s, has visited more than 60 countries and promoted the Chinese traditional music culture throughout the whole country, aiming to show the unique art charms of Muqam to people. Nowadays people seem to forget the core part "Naghma", a fusion of "Western melody" by combining period of China 
Han and Tang dynasties. Thus in current situation protecting and inheriting the originality of Muqam art is more important and challenging than developing it.

\section{CONCLUSION}

We should realize clearly in the land of Xinjiang China, Twelve Muqam Uygur is breaded with its unique art language. It's closely related to national aesthetic consciousness, cultural background and various development stages, which makes it irreplaceable by any other art form. Therefore facing its symphonic question, the answer is very obvious. Its development path should follow its own way and direction. Instead of innovating and striving for changes, making it go farther away from the correct road, we should dedicate ourselves to inherit and develop it to avoid making the wrong decision. In conclusion, our young generation has responsibilities and obligations to let this ancient Chinese art treasures be able to last in our hands.

\section{REFERENCES}

[1] Ji Zhou, Twelve Muqam Uygur Music [M]. Beijing: Central Conservatory of Music Press, March 2008 (in Chinese).

[2] Tingting Wang, Appreciation of Western classical music [M]. Beijing: University of International Business and Economics Press, September 2013 (in Chinese).

[3] Abdushkul Mohamed Yiming, Discussion on the Uygur's classic music Twelve Muqam [M]. Beijing: The Ethnic Publishing House, 1980 (in Chinese).

[4] Jianhua Guan, Yujiang Zhu, Strengthen the exchange of dialogue over the cooperation of world's national music — A summary of the third annual meeting of the World National Music Academy [J]. Music research, 2007, (4):115-120, 123 (in Chinese).

[5] Gulibahar Semti, Research on the protection and tourism development of the world's intangible cultural heritage Uygur Muqam - Taking Yarkant County for example [D]. Xinjiang Normal University, 2016 (in Chinese).

[6] Ji Zhou, Qiu Ci Yi Yun : Discussion on the present inherited relations between Uygur traditional music and Qiu Ci music in Kuqa region [M]. Beijing: Central Conservatory of Music Press, May 2008 (in Chinese). 